\title{
Efforts to Validate the Applicability of Established Chemotherapy Treatment in Turkish Cancer Patients
}

\author{
Turhal NS*, Dane F, Butur S, Kocak M, Telli F, Seber S, Kanitez M, Aktas B and Yumuk PF
}

Marmara University School of Medicine, Department of Internal Medicine, Division of Oncology, Istanbul, Turkey

\begin{abstract}
Introduction: Incidences of cancer in males and females in Turkey are increasing rapidly. However, current incidences are approximately one third of those found in the developed world. In the European Union, Turkey is the sixth largest spender on cancer care, surpassed only by Spain, England, Italy, France, and Germany. The purpose of our efforts is that since there is much variability in- between different societies, based on drug dosing, and administration protocols solely on the experience of the developed world may be misleading. Therefore since there are various differences including pharmacodynamics, pharmacogenomics, toxicity, and efficacy, the each in every society should carry on their own observations on applicability of various chemotherapeutic agents, and combinations, and should set the standards of their own society based on these experiences.
\end{abstract}

Material and methods: Outcome of our patients treated for Breast, Lung and Colorectal cancer with standard treatment protocols are studied for tolerability and outcome. Two-sided statistical tests were performed on the DFS, OS, and toxicities. Survival curves were estimated by the Kaplan-Meier method. Records of patients who were lost to follow-up or alive at the time of analysis were censored at the last documented visit. The differences in DFS and OS were evaluated using log-rank test, or an unadjusted proportional hazards regression model to assess hazard ratios and their $95 \% \mathrm{Cls}$.

Results: The tolerability of the studied chemotherapeutic agents and the patient outcome are comparable to what has been reported in reference studies. But there are some considerable differences that need to be taken into count and these differences are discussed in the text.

Conclusion: There was considerable variability in tolerance and toxicity of chemotherapeutic agents because of pharmacodynamic, and pharmacogenomic variability in Turkish society. Therefore efforts should continue to find easier application schedules, ways and means in countries with less limited resources in cancer care.

Keywords: Chemotherapy; Breast cancer; Colorectal cancer; Renal cell cancer; Lung cancer; Anthracycline; Cisplatin

\section{Introduction}

Marmara University, which was established in 1883 during the Ottoman Empire, was a pioneer of structured medical education. Marmara University Hospital was established much later in 1983 as a 450-bed hospital located in Kaynarca, Istanbul [1]

Incidences of cancer in males and females in Turkey are increasing rapidly. However, current incidences are approximately one third of those found in the developed world. Because of rapid increases in life expectancy in Turkey, the incidence of cancer also is expected to increase rapidly. The most common cancer in men is, as expected, lung cancer with an incidence of close to 70 cases per 100,000 males, followed by prostate cancer (30 cases per 100,000 males), and then bladder cancer (21 cases per 100,000), colorectal cancer (18 cases per $100,000)$, and gastric cancer (14 cases per 100.000). The remaining cancer cases are seen in fewer than 10 cases per 100,000 male populations. For females, breast cancer is common with an incidence close to 40 cases per 100,000 followed by colorectal cancer ( 12.5 cases per 100,000) and thyroid cancer (a little over 11 cases per 100,000). All other cases are seen in less than 10 per 100,000 of Turkish population. Annual antineoplastic and immune modulator use in Turkey was over 4 million boxes as of 2008. Only five years ago this number was about 1.5 million boxes. Thus, in the European Union, Turkey is the sixth largest spender on cancer care, surpassed only by Spain, England, Italy, France, and Germany $[2,3]$.
The purpose of our efforts is that since there is much variability in between different societies, based on drug dosing, and administration protocols solely on the experience of the developed world may be misleading. Therefore since there are various differences including pharmacodynamics, pharmacogenomics, toxicity, and efficacy, the each in every society should carry on their own observations on applicability of various chemotherapeutic agents, and combinations, and should set the standards of their own society based on these experiences.

A variety of factors, including drug dosing and administration protocols can vary widely among countries. Consequently, studies of the factors that affect incidences of cancer conducted in developed countries may not apply in Turkey.

${ }^{*}$ Corresponding author: Prof. Nazım Serdar Turhal FACP, Director, Division of Oncology, Marmara University Research and Training Hospital, Medical Oncology Department Fevzi Cakmak Mah. Mimar Sinan Cad. No 41. Ust Kaynarca Pendik 34890 Istanbul Turkey, Tel: 009021635802 38; Fax: 0090 21635802 37; E-mail: turhal@superonline.com

Received October 15, 2011; Accepted December 06, 2011; Published December 08, 2011

Citation: Turhal NS, Dane F, Butur S, Kocak M, Telli F, et al. (2011) Efforts to Validate the Applicability of Established Chemotherapy Treatment in Turkish Cancer Patients. Pharm Anal Acta S14. doi:10.4172/2153-2435.S14-001

Copyright: ( 2011 Turhal NS, et al. This is an open-access article distributed unde the terms of the Creative Commons Attribution License, which permits unrestricted use, distribution, and reproduction in any medium, provided the original author and source are credited. 


\section{Methods}

Outcome of our patients treated for Breast, Lung and Colorectal cancer with standard treatment protocols are studied for tolerability and outcome. Two-sided statistical tests were performed on the DFS, OS, and toxicities. Survival curves were estimated by the Kaplan-Meier method. Records of patients who were lost to follow-up or alive at the time of analysis were censored at the last documented visit. The differences in DFS and OS were evaluated using log-rank test, or an unadjusted proportional hazards regression model to assess hazard ratios and their 95\% CIs. Proportional hazard regression models of DFS and OS were used to identify significant prognostic covariates. A $\mathrm{p}$ value $<0.05$ was accepted as significant.

\section{Breast cancer}

Marmara University Medical Oncology Department was established in 1997. To date, we have closely monitored a little over 1,000 patients who have been diagnosed with breast cancer. The median age of these patients is 51 . Approximately $54 \%$ of them are postmenopausal; $5 \%$ of them are young (age less then 35 ) breast cancer patients. Size of the tumor at presentation: $52 \%$ of our patients had T2 tumors and $40 \%$ of them had T1 tumors. Multifocality was seen in $16 \%$ of the patients; $53 \%$ of the patients were at Stage 2 and the remainder were equally distributed to Stage 1 and Stage 3. The results of histological observations showed that $78 \%$ of patients had invasive ductal carcinoma, $18 \%$ of the patients had lobular carcinoma, and $14 \%$ of the patients had mixed tumors [4]. The histological status of our patients are similar to that has been described in US literature [5]. Some patients received an adjuvant treatment; $54 \%$ of patients received an anthracycline-based regimen and $20 \%$ of the patients had taxane-based treatment added to their anthracycline-based regimens. In general, $13 \%$ of our patients had recurrences and $5 \%$ were dead. Therefore our analysis for disease-free survival and overall survival had a sufficient number of events to make it valid. A review of survival outcomes showed that the 10-year overall survival rate and the 10-year disease-free survival rate, respectively, were $98 \%$ and $85 \%$ for Stage 1 patients, $79 \%$ and $85 \%$ for Stage 2 patients, and $65 \%$ and $67 \%$ for Stage 3 patients [4].

We evaluated anthracycline tolerance of our patients. Based on personal communications, many physicians had found far higher toxicity than the levels reported in the literature from the developed world. In our data collected from 2006 to 2009, we studied 110 patients. Median age of these patients was comparable to the main group. Median follow-up of these patients was over 2 years. The relative dose intensity rate of our patients was 0.876 , the range was $0.42-1.06$, and these values were not significantly lower than the data from the developed world [5]. We looked to see if there is any association with dose intensity and other factors. We found no evidence of an association of T stage, nodal stage, grade, age, occupation, or comorbidities. The only variable that made a difference in dose intensity was whether patients received a 2-drug regimen or a 3-drug regimen, that is, whether patients received a combination of cyclophosphamide and epirubucin or a combination of cyclophosphamide, epirubucin and 5-fluorouracil. The dose-limiting toxicity was asthenia and gastrointestinal toxicity [6].

\section{Colorectal cancer}

The efficacy, safety and prognostic features of resected colon carcinoma treated in 3 oncology centers in Turkey were studied retrospectively, and published in Balkan Union of Oncology Journal. In the study, the median age of the colorectal cancer patients was approximately a decade older than breast cancer patients; $58 \%$ of the patients were younger than 65 years of age. There was a slight male predominance at $54 \%$. A majority of the lesions (48\%) were localized in the sigmoid colon. A majority of colorectal cancer patients (51.8\%) was in Stage IIIb and 26\% were in Stage IV. In a majority of patients (78\%), the depth of invasion was at the T3 level. The depth of invasion was at the $\mathrm{T} 4$ level in $18 \%$ of patients. The fraction of patients with $\mathrm{N} 1$ nodal involvement and the fraction with no nodal involvement was 54\% and $36 \%$, respectively. The fraction of patients with intermediate grade and high grade tumors, respectively, was $65 \%$ and $20 \%$. The fraction of patients with lymphatic invasion, vascular invasion, and perineural invasion, respectively, was $50 \%, 33 \%$, and $24 \%$. Mucine production was found in $23 \%$ of patients. When we gave adjuvant treatment for these patients, Grade 3 and Grade 4 toxicities were most commonly seen as a form of diarrhea, which occurred in $6.7 \%$ the patients. The other most common toxicity was asthenia, which was seen in $4.2 \%$ of the patients. The overall, five-year survival rate for all patients was $34.8 \%$. The overall, five-year survival rates for patients with 5 -fluorouracil based treatment and for patients in more advanced stages who received both oxaliplatin and 5-fluorouracil based treatment were $42 \%$ and $22 \%$, respectively. Disease-free survival rates at 3 years were calculated for all patients $(69 \%)$, for patients with only 5-fluorouracil-based treatment (67\%), and for patients receiving oxaliplatin, and 5-fluorouracil-based treatment (83\%). The cancer-specific survival rate at 5 years was $78 \%$ for all patients and $77 \%$ for patients who received a 5-fluorouracilbased treatment. Probability of overall survival at 5 years was $73 \%$ for all groups and $72 \%$ for patients who received the 5 -fluorouracil-based treatment. For patients who received oxaliplatin in addition to the 5-fluorouracil-based treatment, the five-year overall survival rate and the five-year cancer-specific survival rate were not available due to the short follow-up for this group. We evaluated effects of individual baseline factors on disease-free and cancer-specific survival but in multivariate analysis only $\mathrm{T}$ stage and perineural invasion had a significant effect. We found no evidence of a significant effect of age, gender, localization, tumor diameter, disease stage, end-stage, lymphatic invasion, vascular invasion, mucine production, grade and total number of lymph nodes dissected. When we looked at the tolerability of the 5-fluorouracilbased chemotherapy group, $52 \%$ of the patients had a dose reduction because of toxicity and $73 \%$ of the patients were given the total planned dose and the total number of cycles of chemotherapy. For the group of patients who received oxaliplatin in addition to 5-fluorouracil, $18.5 \%$ of the patients had a dose reduction because of toxicity and $66 \%$ of the patients were given the total planned dose and the total number of cycles of chemotherapy ( $\mathrm{p}=0.56$ for dose reduction, and $\mathrm{p}=0.44$ for completeness of the treatment schedule between the 5-fluorouracilbased treatment group and the oxaliplatin+5-fluorouracil-based treatment group). Overall $16.5 \%$ of the patients had a dose reduction because of treatment-related toxicity and $70 \%$ were given the total planned dose and the total number of cycles of chemotherapy. More than $90 \%$ of the chemotherapy dose was actually given [7].

\section{Lung cancer}

We also looked at the tolerability of different schedules of chemotherapy using a combination of platinum and vinorelbine 
in advanced non-small cell lung cancer. The recommended dose of vinorelbine $\left(30 \mathrm{mg} / \mathrm{m}^{2}\right)$ was given on days 1,8 , and 15 and the recommended dose of cisplatin $\left(120 \mathrm{mg} / \mathrm{m}^{2}\right)$ was given on day 1 . The cycle was repeated every 28 days. For the combination of vinorelbine and carboplatin, vinorelbine $\left(25 \mathrm{mg} / \mathrm{m}^{2}\right.$ iv) was given on days 1 and 8 , and carboplatin with AUC of 8 was given on day 1 . The cycle was repeated every 28 days. We evaluated the efficacy of a lower dose and three weekly chemotherapy schedules. Between 1997 and 2007, we treated 353 patients with Stage IIIb or Stage IV non-small cell lung cancer. Of these patients, 79 received a combination of vinorelbine and platinum. All patients were chemotherapy-naive and had a performance status of 0 to 2 . Vinorelbine $\left(25 \mathrm{mg} / \mathrm{m}^{2}\right)$ was given on days 1 and 8 . Cisplatin $\left(80 \mathrm{mg} / \mathrm{m}^{2}\right)$ or carboplatin AUC 5 was given on day 1 every three weeks. Demographic features of the patients showed that their median age was 60 . The fraction of patients who were younger than age 65 and who were male was $77 \%$ and $89 \%$, respectively; $62 \%$ of patients had 30-60 pack-years of smoking history. Weight loss greater than $10 \%$ was present in $33 \%$ of the patients. For $67 \%$ and $25 \%$ of patients, respectively, performance status was assigned values of 0 and 1 . Of all patients, 31\% had reached Stage III B and the remainder had reached Stage IV. Based on histology, patients were diagnosed with adenocarcinoma (42\%), squamous cell carcinoma (30\%), non-small cell lung cancer not otherwise specified (23\%), large cell lung cancer (5\%). Disease progressed in $85 \%$ of all patients. Overall response rate was $19 \%$. Median time to progression was 3 months. When we looked at the various factors for effect on time to progression (defined as appearance of a new metastatic foci or progression of an existing foci more than $10 \%$ in bi-dimensional measurement), gender, histology, performance status, age, and stage were not significant. The 1-year and 3-year overall survival rates were $45 \%$, and $25 \%$, respectively. Median overall survival was 13 months. There was no evidence of an effect on overall survival of age, gender, histology, performance status, and stage. Median follow-up time was 11 months. A univariate analysis was used to evaluate effects on overall survival of factors such as gender, age, performance status, histology, and stage. None of these factors had a significant impact on overall survival. When we looked at the other non-small cell lung cancers that received chemotherapy regimens different from the combinations of vinorelbine and carboplatin, 2-year overall survival was highest in the group in which patients received a combination of docetaxel and platinum. Survival was similar to the docetaxel-platinun group in the group in which patients received gemcitabine-paclitaxel-platinum but survival was lower in the group in which patients received vinorelbine. A total of 353 patients were analyzed. However, when we looked at them statistically, there was no statistically significant difference. When we looked at the vinorelbine-platinum combination reported earlier in the developed world, our response rate was lower than the other groups, but overall survival, and 1-year overall survival, although not statistically significant, were higher than the levels reported in previous studies. In conclusion, treatment with vinorelbine on day 1 and with platinum every 3 weeks might be a reasonable alternative to a 4-week schedule in terms of median time to progression and overall survival. Results are comparable to results reported previously in the literature. Lower response rate might be secondary to combining the date of the patients who received cisplatin or carboplatin with vinorelbine or administering a lower dose of platinum than the dose that has been reported in the developed world [8].

\section{Cisplatin}

Renal toxicity and quality of life in patients treated with cisplatinbased chemotherapies also were studied in 49 patients; 47 of these patients received the first-line cisplatin-based chemotherapy for lung cancer. Cisplatin dose was $62-100 \mathrm{mg} / \mathrm{m}^{2}$ infused over 90 minutes with mannitol and routine premedication. No overnight hydration was given, no electrolytes were given, and there was no interim blood analysis on these patients. In the study patients, we checked EORTC, QLQ, C- ${ }_{30}$, QoL analyses before, and after Cycle 3. Creatinine values were checked after Cycles 1, 2, and 3, and 6 weeks after Cycle 3, and there were no significant change. Cystatin- $\mathrm{C}$ values were also studied in a similar fashion but no significant difference was noted. Magnesium levels were significantly depleted in cisplatin-based chemotherapy but it was not affected in our patients. GFR was checked before and after Cycles 1 and 3 but there was no statistically significant difference. Our results show that there was no cisplatin-based persistent renal toxicity with the way we administered cisplatin. There was no change in either GFR or electrolyte imbalance. There was no statistically significant change in the quality of life, either [9].

\section{Renal cell cancer}

Sunitinib tolerance was evaluated in 102 patients in three oncology centers in Turkey. The mean age of the patients was 58; 66 of the patients were males; Eighty-four percent of patients had clear cell histology and Fuhrman Grade III and Grade IV disease were seen in $28 \%$ of the patients. Sixty percent of the patients received interferon therapy as the first-line therapy, $34 \%$ received sunitinib as the first-line, and $64 \%$ received sunitinib as second-line therapy. Only $2 \%$ received sunitinib as the third-line therapy. Sixty percent of patients received $37.5 \mathrm{mg}$ of sunitinib per day; $32 \%$ of patients received $50 \mathrm{mg}$ a day 4 weeks out of 6 weeks and $8 \%$ of patients received $25 \mathrm{mg}$ a day. Grade 3-4 side effects included asthenia ( $7 \%$ of patients), followed by diarrhea and hand-foot syndrome (3\% of patients). The remaining side effects were seen in $2 \%$ of patients. Major treatment complications were as follows. Hypothyroidism that required treatment occurred in $28.4 \%$ of patients. Dose adjustment was needed in $40 \%$ of the patients. Dose delay was seen in $25 \%$ of patients. Sunitinib response in stable disease was seen in $52.9 \%$ of patients. Progressive disease was observed in $24.5 \%$ of patients and partial remission in $19.6 \%$ of patients. Survival analysis showed that median survival of these patients was 25 months. One year overall survival was $82 \%$, 2-year overall survival was $57-58 \%$. The median, progression-free survival was 11.7 months [10].

\section{Results}

The tolerability of the studied chemotherapeutic agents and the patient outcome are comparable to what has been reported in reference studies. But there are some considerable differences that0020need to be taken into count as discussed above.

In conclusion, there was considerable variability in tolerance and toxicity of chemotherapeutic agents because of pharmacodynamic, and pharmacogenomic variability in Turkish society. Therefore efforts should continue to find easier application schedules, ways and means in countries with less limited resources in cancer care.

\section{References}

1. Marmara University Official website: General information about Marmara University. 
Citation: Turhal NS, Dane F, Butur S, Kocak M, Telli F, et al. (2011) Efforts to Validate the Applicability of Established Chemotherapy Treatment in Turkish Cancer Patients. Pharm Anal Acta S14. doi:10.4172/2153-2435.S14-001

2. The Ministry of Health of Turkey: The Cancer Control Department: Cancer Registry in 8 provinces.

3. The Ministry of Health of Turkey. E library: Turkish Health Statistics 2010.

4. Basaran G, Devrim C, Caglar HB, Gulluoglu B, Kaya H, et al. (2011) Clinical outcome of breast cancer patients with $\mathrm{N} 3 \mathrm{a}$ ( $\geq 10$ positive lymph nodes) disease: has it changed over years? Med Oncol 28: 726-732.

5. Early Breast Cancer Trialists' Collaborative Group (EBCTCG) (2005) Effects of chemotherapy and hormonal therapy for early breast cancer on recurrence and 15-year survival: an overview of the randomised trials. Lancet 365: 1687-1717.

6. Turhal NS, Dane F, Seber S, Korkmaz T, Yumuk F, et al. (2010) Tolerability of anthracyclines in Turkish breast cancer patients. BUON Congress 2010 , Proceedings Book, 8-11 September 2010, Sibiu, Romania. Oral presentation 32: 14
7. Dane F, Akif Ozturk M, Gumus M, Guven A, Aliustaoglu M, et al. (2011) Efficacy, safety and prognostic features of resected colon carcinoma treated in"real world" practice: a retrospective cohort-study. J BUON 16: 257-264.

8. Yumuk PF, Teomete M, Dane F, Cabuk D, Basaran G, et al. (2008) Platinum plus vinorelbine (VNR) on days 1 and 8 in advanced non-small cell lung Cancer: Single center results. $7^{\text {th }}$ Congress of Balkan Union of Oncology (BUON) Kuşadası Proceedings OP-72.

9. Tezcan S, Izzettin FV, Turhal S (2011) Investigation of Cisplatin Nephrotoxicity in Out Patients Using A Short Hyration Method. The 2011 European Multidisciplinary Cancer Congress Abstract Book 244-245.

10. Kocar M, Turhal NS (2010) Hypothyroidism More Common in Turkish Metastatic Renal Cell Cancer Patients Treated with Sunitinib. Turkish Oncology Group meeting proceedings. 